\title{
ReESTRuCTURACión De LA Agricultura E INSEGURIDAD ALIMENTARIA. LAS INICIATIVAS Y RETOS DE LOS PEQUEÑOS PRODUCTORES EN CHIAPAS
}

\author{
Agriculture Restructuration and Food \\ INSECURITY. INITIATIVES AND CHALlENGES \\ of the Small Producers in Chiapas
}

\author{
Héctor B. Fletes Ocón ${ }^{1}$ \\ Guadalupe Ocampo Guzmán \\ Guillermo Valdiviezo Ocampo
}

Resumen: Organismos internacionales y especialistas coinciden en que los problemas de crisis e inseguridad alimentaria se asocian con las políticas que diferentes países aplicaron en las últimas dos décadas, las cuales desmantelaron los programas de fomento a la agricultura, en particular de los pequeños productores. Aunque esta reestructuración neoliberal se realizó de manera heterogénea, los retos para reactivar la producción agroalimentaria sustentable y de calidad, así como de elevar los ingresos rurales, son de gran magnitud, y en ocasiones no aparecen en los objetivos de la política nacional. En este documento ${ }^{2}$

\footnotetext{
${ }^{1}$ Profesores de tiempo completo de la Universidad Autónoma de Chiapas, Facultad de Ciencias Sociales, San Cristóbal de Las Casas, Chiapas, México. Correos electrónicos: hctrfo@gmail.com,guzocamgua@hotmail.com,gsov67@gmail.com

${ }^{2}$ El texto se basa en resultados preliminares del proyecto "Competitividad agroindustrial y desarrollo territorial en el Pacifico mexicano" —financiado por Prodep Redes Académicas-, desarrollado por la Universidad Autónoma de Chiapas, Universidad de Guadalajara y Universidad Autónoma de Nayarit. En su segunda etapa (2015-2016) el proyecto se enfoca en las Estrategias para seguridad y soberanía alimentaria en el Pacífico mexicano. En el caso de Chiapas, se estudia la región de Comitán, y se ha contado con la participación de los alumnos Carlos $S$. Roblero López, Estefanía I. del Solar Namindamo, Lily Cruz Gómez, Ilse Morelia Trujillo, y Guadalupe Huet Bolom, a quienes se agradece su apoyo.

Fecha de recepción: 2208 16; Fecha de aceptación: 221116.
}

(oc) EY-No-ND Páginas 112-135. 
Héctor B. Fletes Ocón

Guadalupe Ocampo Guzmán

Guillermo Valdiviezo Ocampo

analizamos la forma en que la reestructuración ha impactado en ciertos patrones de creciente inseguridad alimentaria en México y Chiapas, así como la manera en que pequeños productores han adaptado y retado este proceso, constituyendo iniciativas con las que revalorizan la lógica de la agricultura campesina y de pequeña escala.

Palabras clave: política neoliberal, agricultura, seguridad alimentaria, Chiapas.

Abstract: International organisms and specialists agree that crisis and food insecurity are associated to neoliberal policies applied by that different countries in the last two decades. This policies have dismantled prom otion of agriculture, particularly those in favor of small scale producers. Although neoliberal restructuring has had a heterogeneous application, the challenges to reactivate sustainable and quality food production, and to raise rural incomes, are generally vast, and sometimes do not appear in the national policy objectives. In this paper, we analyze the way that this restructuring has impacted in certain aspects of food insecurity in Mexico and the state of Chiapas, and the ways in which small producers have adapted and challenged this process, initiatives with initiatives that revalue the peasant and small scale production logic.

Keywords: neoliberal policy, agriculture, food security, Chiapas.

\section{Introducción}

La historia del desarrollo ha demostrado que la agricultura y alimentación constituyen un sector estratégico para los Estados nación. Igualmente, el estímulo de la producción por pequeños productores (PP) ha sido una esfera permanente de intervención. Este enfoque se modifica por las distintas correlaciones de fuerzas que convergen en tales Estados, y los diferentes principios políticos y de bienestar que los alientan. En México, se emprendió desde hace poco más de dos décadas el camino de la restructuración política neoliberal, que, sin reconocer el amplio 
Héctor B. Fletes Ocón

Guadalupe Ocampo Guzmán

Guillermo Valdiviezo Ocampo
Reestructuración de la agricultura

e inseguridad alimentaria...

margen de asimetrías tecnológicas, económicas y naturales entre los agricultores, condujo al desmantelamiento de un complejo institucional que operaba en respaldo de ese sector estratégico, tanto en términos económicos, como culturales y sociales. Aunado a los fenómenos asociados con el cambio climático, las políticas y programas en este periodo han generado condiciones de alta vulnerabilidad alimentaria local, misma que se acentúa en zonas rurales y en el sector de pequeños productores campesinos (Holt-Giménez y Patel, 2012; Calva, 2012 González, 2013; Fletes y Lozano, 2015).

A mediados de la primera década del siglo XXI, desde diversos foros e instituciones nacionales e internacionales - FAO, Banco Mundial, y Estados nacionales - se presenta un retorno a la consideración de la importancia del fomento de la agricultura como parte de la estrategia de desarrollo nacional, así como el reconocimiento del papel que desempeña la producción de pequeña escala, no sólo en aspectos económicos - abastecimiento local de alimentos e ingresos-, sino también sociales y ambientales (Fletes et al., 2014a). Los temas de la inseguridad y vulnerabilidad alimentaria adquieren una gran relevancia en el contexto de la crisis económica y de alimentos de finales de esa década (Rubio, 2014). Sin embargo, considerando las condiciones anteriores — procesos a los que habría que agregar la emigración rural, el abandono de actividades agropecuarias, el debilitamiento del tejido social rural-, se encuentra que los retos para reactivar la producción agroalimentaria sustentable y de calidad son de gran magnitud.

En ese sentido, como actores participantes en los procesos de cambio rural y agroalimentario, nos encontramos actualmente en la necesidad de reflexionar y actuar para el rediseño institucional que tenga como estrategia fundamental la seguridad y soberanía alimentarias, con base en pequeños productores, en términos de equidad y sostenibilidad. Sucede que los principios de política recientemente aplicados bajo los términos de eficiencia, mercado libre, competitividad y rentabilidad, no son neutrales, sino que se construyen en un marco de relaciones de desigualdad y poder, y conllevan impactos sociales, culturales, ambientales y económicos, de gran calado (Fletes, 2013). Estos fenómenos se exacerban en regiones rezagadas del país, como es el caso de Chiapas, 
dadas sus conocidas condiciones de pobreza, dispersión poblacional, estrechez del patrón productivo y bajo valor agregado de los productos (Villafuerte, 2015).

Por lo anterior, en el presente documento, analizamos la forma en que la reestructuración económica y política ha impactado en ciertos patrones de creciente inseguridad alimentaria en México y Chiapas, así como la manera en que pequeños productores en la región chiapaneca de Comitán han enfrentado, adoptado y retado este proceso. Para ello, en el siguiente apartado analizamos los principales planteamientos ideológicos que dieron pie a la reestructuración política y económica en el país, y algunos de sus efectos. En el tercer apartado, se ofrece un panorama de elementos relacionados con la inseguridad alimentaria en Chiapas, con énfasis en la región de Comitán. Posteriormente, presentamos un panorama de las iniciativas que están desarrollando los productores, con las que revalorizan la lógica e importancia de las prácticas de la agricultura de pequeña escala. El documento concluye con algunas reflexiones entorno al tema de estudio.

\section{Reestructuración política en relación con los pequeños productores en México}

En México, en el trascurso de los últimos 100 años, a las unidades pequeñas de producción se las ha visto como un lastre, un impedimento para el desarrollo del campo mexicano. Con base en el Censo Agrícola de Inegi del año 2007, se sabe que a pesar de las distintas disposiciones legales sobre el tamaño mínimo de la parcela, y de la ausencia de políticas dirigidas al desarrollo del minifundio, la de los pequeños productores es la unidad de producción más generalizada en el campo mexicano. En ese año existían 2.68 millones de unidades de producción con menos de cinco hectáreas, que representan $71.6 \%$ del total. Estas unidades de producción (UP) se han multiplicado y han resistido tales disposiciones y la falta de apoyo gubernamental (Robles, 2012).

En el país, desde la década de 1990 se instrumentaron medidas como el control de gasto público, la privatización de numerosas empresas públicas y la eliminación o fusión de organismos, empresas e instituciones. En materia agropecuaria el número de entidades públicas 
en 1982 era de 102, y subsistían 27 en el año 2006 (Lechuga, 2006). Ante el desmantelamiento y privatización de entidades paraestatales vinculadas al sector agropecuario - como Conasupo, Fertimex y Banrural - el vacío fue parcialmente llenado por grandes compañías transnacionales: Cargill, Archer Daniels Midland, Bunge y Dreyfus (Tetreault, 2012).

Los aspectos fundamentales de la ideología que llevó a las acciones que se desarrollaron desde esta década fue por un lado la fe en las fuerzas del mercado para asignar de manera eficiente los recursos entre los agentes económicos y producir el bienestar general, y por el contrario la creencia de que la intervención del Estado distorsionaba este equilibrio y eficiencia. De manera concreta, se consideró que los subsidios distorsionaban los precios, y que los recursos deberían concentrarse en las unidades más eficientes. En ese sentido, por un lado se cambia la estrategia de seguridad alimentaria — se obtendría alimento "barato de cualquier lugar" - y, por otro, los recursos se dirigen a unidades de gran escala y empresariales, dejando de lado a las unidades campesinas y de pequeña escala, que fueron consideradas como población atendible con programas sociales.

En general, comenzó un proceso de desintegración de cadenas alimentarias en México directamente relacionado con el Tratado de Libre Comercio de Norteamérica (Moreno-Brid y Ros, 2010), acompañado de un debilitamiento de la participación de los pequeños agricultores y residentes rurales que solían integrar algunos nodos productivos. La reestructuración ha generado una desconexión entre producción de alimentos e industria alimentaria. La producción se concentra en sectores más rentables como las frutas y hortalizas, pero no en granos que en una fracción significativa son importados. La especialización ha generado una exclusión del mercado de un gran número de pequeños y medianos productores, mientras que el déficit comercial en alimentos crudos y procesados se ha incrementado (Otero et al., 2013).

La incapacidad del modelo basado en el TLCAN de generar suficientes fuentes de empleo y asegurar la seguridad alimentaria ha llevado a varios autores a cuestionar el enfoque de las ventajas comparativas (Puyana y Romero, 2008; Turrent et al., 2012; Fletes et 
al., 2014a). De 1991 a 2007, hubo 4.9 millones de familias desplazadas de agricultores mexicanos; mientras que el trabajo estacional en las industrias de agroexportación se incrementó en cerca de 3 millones. Esto significó una pérdida neta de 1.9 millones de puestos de trabajo (Weisbrot et al., 2014). Igualmente, los altos y crecientes precios internacionales recientes hacen demasiado caro el enfoque de las ventajas comparativas, o el de "precios únicos" (Turrent et al., 2012; Puyana y Romero, 2008).

Durante el proceso de restructuración los agricultores mexicanos enfrentaron una caída en los precios de los cultivos tradicionales. Si bien esa tendencia se remontaba a décadas anteriores, fue acentuada por la apertura y tenía un impacto devastador sobre los ingresos de los pequeños productores. El impresionante incremento en la producción y exportación de frutas y verduras desde entonces ha sido concentrado en las manos de los grandes y medianos productores vinculados a las corporaciones transnacionales. Los campesinos han sufrido un aumento en los costos de la producción, la desaparición de crédito y el retiro de apoyos a la comercialización. Bajo estas condiciones, la actividad agropecuaria de pequeńa escala ha perdido rentabilidad (Tetreault, 2012). La mayor parte de las unidades de producción a pequeña y mediana escala operan a menos de $50 \%$ de su potencial (Turrent et al., 2012). Los programas recientes orientados a pequeńos productores están fragmentados, y finalmente favorecen a grandes compañías agroalimentarias, algunas de ellas transnacionales. En ese sentido tienen un carácter regresivo y han generado mayor desigualdad en el sector rural: se concentran en estados y agricultores con condiciones de mayor capitalización, mientras que en el caso de los estados más pobres no se fomentan las actividades productivas sino apoyos de combate a la pobreza (Robles, 2013).

De acuerdo con lo anterior, los problemas principales en torno a los pequeños productores (PP) consistirían en:

1. La mayor parte del presupuesto Estatal se destina a los denominados "productores con potencial productivo" — grandes propietarios y agroindustrias—, que no son precisamente los más pobres, productores de pequeña escala, la gran mayoría. 
2. El desplazamiento de los programas productivos por programas sociales, los cuales tienen mayor cobertura en zonas rurales (Robles, 2012; Fletes et al., 2014b).

3. El privilegio obtenido por la agricultura industrial, lo cual contribuye fuertemente en la degradación de los recursos naturales del país.

Una de las distorsiones de ver la agricultura campesina y de PP como "tradicional" es no tomar en cuenta la gran variedad de sistemas y grados de intensidad que puede tomar, según las condiciones internas y externas de la unidad doméstica. En este debate es crucial entender la racionalidad de los pequeños productores campesinos para comprender las condiciones que conducen a su productividad, su diversidad y sus formas particulares de organización (Nigh, 2010).

A pesar de la posición subordinada que se le ha asignado en las prioridades políticas, la agricultura —en particular de PP y campesinosse constituye en un espacio desde donde se gestan nuevas alternativas para enfrentar, adoptar y/o resistir las condiciones arriba señaladas. Las organizaciones campesinas mexicanas —agrupadas en el movimiento "El campo no aguanta más", y después en "Sin maíz no hay país" - articularon en los años 2002 y 2003 "Seis propuestas para la salvación y revaloración del campo mexicano”, en las cuales se pretendía recuperar algunos de los mecanismos proteccionistas empleados en el pasado, e implicaba la necesidad de renegociar el TLCAN —desde el año 2013, el Frente Autentico del Campo aglutina a las principales organizaciones campesinas del país- Al establecer bajo el concepto de soberanía alimentaria el acceso a los alimentos como un derecho humano - lo cual fue retomado por el Estado mexicano en el año 2011, mediante la modificación de la Constitución Política para que éste garantice el derecho a la alimentación nutritiva, suficiente y de calidad - se propone un proceso de valorización de la pequeña agricultura, y una transición a la agroecología (Tetreault, 2012; Rodríguez-Gómez, 2013; McMichael, 2015).

La soberanía alimentaria es una propuesta de la Vía Campesina (VC), movimiento internacional que agrupa 150 organizaciones campesinas locales y nacionales en 70 países de África, Asia, Europa 
y América, siete ellas de México. Las organizaciones que forman parte del movimiento comparten un rechazo al modelo neoliberal, demandan participar en la formulación de políticas agropecuarias y están determinadas a establecer un modelo alternativo de agricultura.

Mediante el concepto de soberanía alimentaria, VC demanda responsabilidad al Estado - a diferencia del concepto de seguridad alimentaria, que se concentra en la disponibilidad de alimentos, sin importar de donde provengan - para coordinar una estrategia de producción agropecuaria orientada a múltiples objetivos, entre ellos: la seguridad de tener suficientes alimentos básicos para la población nacional, la producción de comida sana, sabrosa y culturalmente apropiada, la superación de la pobreza rural y la protección del medio ambiente (Tetreault, 2012; Edelman, 2014).

\title{
Aspectos de inseguridad alimentaria en Chiapas y la región de Comitán ${ }^{3}$
}

A pesar del debate en distintos espacios académicos y foros, en el tiempo ha predominado la concepción de la FAO sobre inseguridad alimentaria, entendida como la

\begin{abstract}
Situación que se da cuando las personas carecen de acceso seguro a una cantidad de alimentos inocuos y nutritivos suficiente para el crecimiento y desarrollo normales así como para llevar una vida activa y sana. Las causas son múltiples: no disponibilidad de alimentos, poder adquisitivo insuficiente, distribución inapropiada o uso inadecuado de los alimentos en el interior del hogar (FAO, 2015: 58).
\end{abstract}

\footnotetext{
${ }^{3}$ En este trabajo consideramos la región de Comitán en los términos del Distrito de Desarrollo Rural que maneja la Sagarpa. Esto nos permite hacer comparaciones de los cambios productivos en un periodo amplio, principalmente para años previos a 2011 cuando el gobierno del estado de Chiapas modifica la región político-administrativa que manejaba hasta entonces. Igualmente, nos deja ver relaciones concretas de carácter social y productivo que ocurren entre los actores de este estudio. El Distrito de Comitán se compone de los siguientes municipios: Chicomuselo, Comitán, Frontera Comalapa, La Independencia, La Trinitaria, Las Margaritas, Maravilla Tenejapa, Socoltenango y Tzimol.
} 
Héctor B. Fletes Ocón

Guadalupe Ocampo Guzmán

Guillermo Valdiviezo Ocampo
Reestructuración de la agricultura e inseguridad alimentaria...

Un aspecto criticable de esta noción lo plantean González y Macías (2007), al afirmar que el concepto de seguridad alimentaria es ambiguo, y sólo hace referencia a la disponibilidad de los alimentos sin considerar la producción de los mismos. Defienden que ambos elementos — producción y disponibilidad de los alimentos- son interdependientes y están condicionados por factores ambientales; de ahí que deban ser explícitamente tomados en cuenta. De tal modo que proponen incorporar el concepto de vulnerabilidad alimentaria, que se entiende como "la situación que caracteriza a países, sectores sociales, grupos e individuos que están expuestos o son susceptibles de padecer hambre, desnutrición o enfermedad por no tener acceso física, económica y sustentablemente a una alimentación suficiente, nutritiva y culturalmente aceptable, o por consumir productos insalubres o contaminados" (íbid.).

Esta vulnerabilidad puede presentarse en forma progresiva y constante, como la erosión del suelo, la desertificación, el cambio climático, la disminución de la biodiversidad de los cultivos. O de manera súbita, como un cambio en las políticas agroalimentarias que mine la capacidad productiva de un país y lesione el poder adquisitivo y la capacidad de recuperación de amplios sectores de su población.

La inseguridad alimentaria se relaciona tanto con la producción de alimentos como con la pobreza. En México, esta asociación se muestra en los resultados presentados por el Consejo Nacional de Evaluación de la Política de Desarrollo Social (Coneval) en los distintos informes desde el año 2010 a 2014 . Aunque ha disminuido la pobreza extrema — de 11.3 a $9.5 \%$ de la población-, el resultado de carencia por acceso a la alimentación mantiene un elevado porcentaje - de 24.8 a 23.4\% de la población; esto es, de 28.4 a 27.9 millones de personas-, situación que tiene que ver con la aplicación de programas sociales en los sectores de extrema pobreza, pues elevan en alguna medida el nivel de ingresos, pero no resuelven el problema alimentario al no enfocarse en la producción.

En el caso de Chiapas, los niveles de ingresos son claramente insuficientes para la adquisición de los alimentos básicos (ver Tabla 1 en siguiente página): 
Tabla 1. Población de Chiapas con ingresos inferiores a las líneas de bienestar, 2010-2014.

\begin{tabular}{|c|c|c|c|c|}
\hline \multirow{2}{*}{ Año } & \multicolumn{2}{|c|}{$\begin{array}{c}\text { Población con ingreso inferior } \\
\text { a la linea de bienestar minimo } \\
\text { (cobertura de alimentación) }\end{array}$} & \multicolumn{2}{|c|}{$\begin{array}{c}\text { Población con ingreso inferior } \\
\text { a la linea de bienestar }\end{array}$} \\
\cline { 2 - 5 } & $\%$ & Personas (miles) & $\%$ & Personas (miles) \\
\hline 2010 & 50.9 & $2,507.1$ & 80.9 & $3,983.0$ \\
\hline 2012 & 46.7 & $2,365.2$ & 76.4 & $3,869.9$ \\
\hline 2014 & 48.5 & $2,518.6$ & 78.7 & $4,090.8$ \\
\hline
\end{tabular}

Fuente: Coneval, 20 de junio de 2016

En Chiapas se presenta una disminución de la producción de alimentos básicos, asociado con el abandono de las actividades agrícolas en las unidades de producción, la penetración y expansión de cultivos destinados a la industrialización, por ejemplo agrocombustibles — palma africana y piñón-, y la proliferación de cultivos comerciales diferentes a los granos básicos, muchos de los cuales fueron promovidos por el propio Estado desde la administración del periodo 2006-2012: limón persa, aguacate, tomate, entre otros.

Eso ocurre en el marco de una estrategia general de desarrollo agrícola llamada "reconversión productiva", que se ha enfocado principalmente en la sustitución del maíz por otros cultivos considerados "comerciales" (Fletes y Bonanno, 2013; Villafuerte, 2015). La lógica de reconversión —que presupone una "baja rentabilidad del maíz" - se mantiene en el sexenio actual, ya que el gobierno del estado anunció que tan sólo en el mes de agosto de 2016 se entregaron 23 mil árboles frutales - limón persa, chicozapote, aguacate hass, guanábana, durazno-- para diversificar la producción (Instituto de Comunicación Social del Estado de Chiapas, 2016).

En la región de Comitán, periodo de 2003 a 2015, la superficie agrícola total cultivada tuvo una drástica caída, pues pasó de $197 \mathrm{mil}$ a 154.2 mil hectáreas, mientras que la de maíz bajó de 139.9 a 100.2 mil ha (SIAP, 2016). 
En este periodo se presentan dos procesos muy relevantes para los agricultores y desde el punto de vista de la intervención del Estado: la disminución de la producción de maíz — también de frijol y café-, y su diversificación en maíz blanco y amarillo, siendo ya más amplia la superficie del segundo; de igual manera se da la diversificación productiva, pues aumenta de 24 a 35 cultivos y variedades, algunos de éstos "nuevos" cultivos, como aguacate hass, cacahuate, cańa de azúcar semilla, chile habanero y verde, limón persa, litchi, durazno, piñón, mandarina, tomate rojo y verde (SIAP, 2016; Herrera, 2016).

Tan sólo la caída de la superficie de maíz significa un problema grave de precaria seguridad alimentaria y vulnerabilidad en la región. Este fenómeno se asocia con las políticas neoliberales, la reconfiguración de las cadenas agroalimentarias y el problema de precios (notas de campo). Cabe mencionar que, a pesar de esta tendencia a la diversificación productiva, es abrumadora la presencia de maíz en las unidades de producción. Por ejemplo, el maíz se encuentra en $88 \%$ de las unidades en Comitán, en 95\% de las de La Trinitaria, y en 100\% de La Independencia.

Esta transformación se acompaña de un grado de polarización agraria y de una gran inestabilidad en la producción. Los mayores porcentajes de superficies agrícolas con riego corresponden a cultivos comerciales que han sido introducidos recientemente. En el año 2003 se regaban 15,142 de las 197,921.8 ha totales, esto es, apenas 7.6\%. Entre los cultivos con riego se encontraba la cańa de azúcar industrial — la totalidad de su superficie—, cebolla — total de superficie—, frijol, maíz grano — 3\% de la superficie total—, melón (total), sandia (total) y tomate rojo variedad rio grande (total), evidenciando que la producción de hortalizas tiene un carácter distinto a la de maíz grano o café. En el año 2015, se regaron 17,498 de las 154.2 miles de ha, esto es, $11.3 \%$ —aun en el contexto de una reducción significativa de las lluvias, escurrimientos y almacenamiento de agua- En este año, se mantiene un patrón de uso de riego en hortalizas y plantaciones, y aparecen nuevos cultivos que son irrigados, como cańa de azúcar semilla, chiles habanero y verde, tomate rojo saladette y maíz blanco, estos últimos con una gran inestabilidad en la superficie de riego. 
Héctor B. Fletes Ocón

Guadalupe Ocampo Guzmán

Guillermo Valdiviezo Ocampo

\section{Transnacionalización regional a través del tomate}

De los cultivos recientes, el que ilustra un grado importante de reconfiguración en la estructura agraria, social y productiva, con nuevos agentes económicos, es el tomate. La presencia en la región comiteca de la empresa Seminis, perteneciente a Monsanto, así como de un grupo de empresas especializadas — distribuidoras de semillas híbridas, productores de plántula, distribuidores de agroquímicos, instaladores de invernaderos, y de sistemas de riego por goteo, etc.- , como son Conagro, Agrocima — antes Plantagro-, da cuenta de la proliferación de verdaderas mercancías alimentarias, cuyas redes productivas y de trabajo conectan con cadenas de distribución que salen de la región e incluso del país. Este fenómeno significativo permite observar un cambio que modifica los recursos bióticos locales - lo cual constituye una de las dimensiones relevantes en regiones conectadas con cadenas transnacionales de mercancías (Linck et al., 2014)—, tecnológicos y la previa "especialización” agrícola en granos.

Con base en el trabajo de campo que realizamos en la región de estudio, se puede identificar desde la década de los sesenta un proceso de "aprendizaje colectivo regional" relativo al tomate, su tecnología, producción y distribución, en donde converge ahora un conjunto heterogéneo de actores participantes en la agroindustria.

En la historia productiva del tomate, resulta relevante la participación de un comerciante del estado de Puebla que en un inicio transportaba tomate de esta región (Gómez, 2013), quien identifica condiciones favorables para ampliar su producción en algunas localidades del municipio y se constituye en un actor central en el desarrollo posterior de la agroindustria.

El grado de desarrollo tecnológico regional a través de este cultivo es significativo debido a la constitución de un complejo agroindustrial de avanzada con el tipo de empresas señaladas. Esto es marcadamente diferente tanto a las características generales de producción agrícola en el estado de Chiapas, como a la propia región de la meseta comiteca, con presencia predominante de una economía campesina, y donde grupos sociales ejercen resistencia a la incorporación de semillas híbridas y a la intensificación de la agricultura mediante la tecnología. 
Héctor B. Fletes Ocón

Guadalupe Ocampo Guzmán

Guillermo Valdiviezo Ocampo

\section{Las iniciativas, límites y retos de los pequeños productores}

En todo proceso de investigación conviene analizar las prácticas de los actores con la finalidad de identificar las maneras en que éstos significan las políticas y las estrategias de ciertos agentes, y con ello crean ciertos márgenes de acción para enfrentar condiciones que les son impuestas, pero que no constriñen su capacidad de creatividad y respuesta. En Chiapas, según el Censo Agrícola de Inegi del año 2007, el promedio de superficie en las Unidades de Producción Rural (UPR) con actividad agropecuaria y forestal fue de 7.5 hectáreas, mientras que en UPR con actividad agrícola exclusivamente, este promedio fue apenas de 5.7 ha. Un indicador relacionado, nos deja ver que las UPR con "consumo familiar" de la producción en Chiapas representan $88 \%$ del total de UPR con superficie agrícola, vivero o invernadero, mientras en la región son $96 \%$. En este sentido, los pequeños productores campesinos representan la inmensa mayoría de las UPR en el estado y región. Cualquier estrategia para estimular su acceso a alimentos debe considerar sus características culturales, de organización económica y de manejo de recursos. Como indica FAO en sus últimos informes:

En todo el mundo en desarrollo, la mayoría de los pobres y las personas que padecen hambre vive en las zonas rurales, donde la agricultura familiar y a pequeña escala es un modo prevalente, aunque no universal, de organización agrícola. Si bien la capacidad de la agricultura familiar y a pequeña escala para estimular el crecimiento a través de una mayor productividad varía notablemente, la función que desempeña para reducir la pobreza y el hambre es fundamental. El crecimiento de la agricultura familiar y a pequeña escala, por medio de un incremento de la mano de obra y la productividad de la tierra, tiene efectos positivos significativos en los medios de vida de los pobres debido a la mayor disponibilidad de alimentos e ingresos que genera (FAO, 2015: 27).

Considerando que la organización económica y de uso de recursos varía conforme al cultivo predominante de que se trate, a continuación analizamos las prácticas de los PP, agrupando por cultivo. 
Héctor B. Fletes Ocón

Guadalupe Ocampo Guzmán

Guillermo Valdiviezo Ocampo
Reestructuración de la agricultura

e inseguridad alimentaria...

\section{Campesinos maiceros}

Además de la desaparición de las entidades que participaban en la provisión de crédito y la comercialización de granos — principalmente Conasupo y Banrural-, la reducción de las superficies de maíz se relaciona con una caída en los precios pagados a los productores, pero también con una reorganización familiar en que los hijos están migrando tanto a otras ciudades del país como a Estados Unidos. Se observa que ante la reducción de la superficie de maíz, lo que hacen las familias en primer lugar es mantener el mayor volumen posible en el hogar para la alimentación de todo el año. Esto es, si se reduce el volumen de maíz obtenido por diferentes circunstancias — siendo ahora la inestabilidad del clima y de las lluvias uno de los principales factores-, se asegura en primera instancia el consumo de la familia. Cuando tienen volúmenes que consideran excedentarios, pueden venderlos a lo largo del año. Pero estas ventas se reducen al mínimo, o se cancelan totalmente, para dejar el mayor volumen en casa. Cabe mencionar que el maíz no es suficiente para autoabastecerse en estas unidades familiares, y muchas de ellas tienen que comprar pequeños volúmenes a lo largo del año. El maíz y otros productos de la región - limón persa, sorgo, mango, tomate- son vendidos, en cierta medida, a comerciantes que llevan el producto al vecino país de Guatemala, aunque de manera muy importante también en el mercado local y regional.

Aunque en la zona de riego de San Gregorio predomina el maíz mejorado o híbrido, la mayoría de los productores de maíz de la región utilizan semillas criollas (Tabla 2). La defensa y persistencia de la semilla criolla de maíz se debe a su mejor resistencia a los cambios del clima, ya que las siembras son de temporal, a su mejor calidad para consumo en el hogar, y a la posibilidad de utilizar varias semillas de acuerdo con las circunstancias del clima. Estos campesinos se constituyen en guardianes de los recursos bióticos locales, pues se encuentra una diversidad de semillas locales, o adaptadas en el tiempo, de maíz, entre las cuales se mencionan: mayero, crema, grano de oro, taxa, Cintalapa, chimbo, tuxpeńo, mopalu, olotillo, Ixhuatán, tehua. Todas con diferentes fechas de siembra. Éste es un trabajo "que los campesinos ofrecen gratuitamente" de conservación de la biodiversidad (Otero, 
Guillermo Valdiviezo Ocampo

e inseguridad alimentaria...

2014: 31), situación que debe valorarse y actuar en consecuencia desde la política pública.

Tabla 2. Prácticas de cultivo y alimentarias desarrolladas por los productores y unidades familiares en el Distrito de Comitán. ${ }^{4}$

\begin{tabular}{|l|c|}
\hline Superficie total promedio & 4.19 ha \\
\hline Superficie promedio de maíz & 1.9 ha \\
\hline $\begin{array}{l}\text { Unidades de producción con maíz que parte es para } \\
\text { autoconsumo }\end{array}$ & $88.6 \%$ \\
\hline Unidades donde algún integrante labora con salario & $14.9 \%$ \\
\hline $\begin{array}{l}\text { Tuvo alguna ocasión sin alimento suficiente } \\
\text { en el último mes }\end{array}$ & $38.6 \%$ \\
\hline Algún integrante con hambre en la semana & $12.3 \%$ \\
\hline Meses con escasez de alimento & $97.4 \%$ \\
\hline Programa social/alimentario & siesenta \\
\hline Número de cultivos & $85.1 \%$ \\
\hline Gasto en alimentos por semana & 2.2 \\
\hline Uso de semilla criolla de maíz & 370.9 pesos \\
\hline Uso de fertilizante químico & $71.9 \%$ \\
\hline Uso de herbicida & $93 \%$ \\
\hline Redujo superficie de maíz en última década & $33.3 \%$ \\
\hline Pertenece a una organización & $38.6 \%$ \\
\hline Familia con inseguridad alimentaria & $21.1 \%$ \\
\hline
\end{tabular}

Fuente: Elaboración propia dentro del proyecto citado

${ }^{4}$ La información se basa en el avance de una encuesta que levantamos para el proyecto citado, aplicada a una muestra aleatoria de 114 hogares rurales y de zonas semiurbanas de la región de estudio. 
Héctor B. Fletes Ocón

Guadalupe Ocampo Guzmán

Guillermo Valdiviezo Ocampo

\section{Campesinos productores de tomate}

Un número reducido de acciones de los productores se lleva a cabo de manera colectiva. En el municipio de La Trinitaria, el grupo Yalplash, una "Sociedad de Producción Rural", se constituyó específicamente para la producción de tomate en invernadero. El grupo se formó en el año 2013, por 30 miembros, de los cuales se retiraron 10 debido a que percibían que la recuperación de la inversión a aportar sería lenta. Es importante resaltar que todos los socios son jóvenes hijos de ejidatarios o de avecindados - los que llegan como pobladores a un asentamiento núcleo del ejido-, pero que formalmente no poseen tierras debido a las características de este régimen de propiedad en México — bajo esta titulación del Estado, y aun con las reformas al art. 27 constitucional del año 1992, los derechos ejidales se transmiten con aprobación de la asamblea ejidal- . En un esfuerzo de gestión que les llevó dos años, y después de tocar distintas puertas, lograron la construcción de dos invernaderos para la producción de tomate, de alrededor de un cuarto de hectárea cada uno, y han obtenido una producción que les es rentable, superior a la que obtenían con maíz.

Antes de formar el grupo, los jóvenes venían cultivando, junto con el maíz, tomate a "cielo abierto", en lotes pequeños. Esto les dotó de cierto conocimiento sobre la producción, proveedores, mercado, además del contexto de aprendizaje regional y de redes sociales, comentado, que les permitió a su vez la incorporación de tecnología de punta como es el propio invernadero, riego por goteo, y uso de variedades tanto "determinadas" como "indeterminadas" — las cuales producen durante un periodo más largo que las primeras-. Un elemento importante de organización es el trabajo colaborativo que realizan de manera programada los jóvenes para la producción de tomate y el mantenimiento de los invernaderos, mientras que la distribución de ingresos se efectúa "de una manera equitativa", teniendo programado para el futuro el establecimiento de otras hortalizas de carácter orgánico. Aunque han encontrado problemas de abastecimiento de agua para el riego en el invernadero. Un aspecto final importante de destacar es que el grupo contribuye al arraigo al territorio, y contrarresta el ya tradicional movimiento poblacional sea a las ciudades o a Estados Unidos. 
Héctor B. Fletes Ocón

Guadalupe Ocampo Guzmán

Guillermo Valdiviezo Ocampo

\section{Organizaciones sociales}

Otro actor de la transformación agraria a favor de los pequeños productores en la región son las organizaciones sociales. Un grupo, vinculado con organizaciones de escala nacional, está orientado a proyectos de desarrollo, sustentabilidad, capacitación, seguridad alimentaria o protección de la biodiversidad, mientras que otros sólo operan para vender los productos por su cuenta de manera directa a los consumidores en mercados de la ciudad de Comitán.

En el primer grupo se encuentra la Organización Campesina Emiliano Zapata (OCEZ-CNPA), compuesta por aproximadamente 5,000 productores en el estado, y cuyas actividades se dirigen principalmente a la seguridad y soberanía alimentaria, agroecología, educación y economía local. Estas organizaciones han ampliado los objetivos que anteriormente tenían, centrados en la lucha agraria; sin embargo, ya estiman que se aproxima una reactivación de este problema social, dado el aumento de población rural. A través de una red que cubre 16 municipios en el estado, gestionan algunos proyectos de desarrollo social y agrícola para los miembros, entre los que se encuentran actividades orientadas a seguridad alimentaria como talleres y unidades de producción en huertos de traspatio diversificados, producción de porcinos y miel.

En cuanto a las organizaciones de comerciantes, se encuentra la de Canasteras y Pequeños Productores Independientes Zapatistas (Capiz), con sede en la ciudad de Comitán, que cuenta con 833 locatarios que a su vez son productores, según señala el líder. Es una forma de lo que se conoce en la literatura como "mercados de productores", que venden entre otros productos elote, frijol, maíz, cacahuate, chayote, naranja, manzana, limón, tomate, cebolla, plátano y quesos. Su formación, en el año 2009, obedeció a la inconformidad de un grupo amplio de productores campesinos de la región con el acaparamiento de la comercialización que realizaban lo que ellos denominan "introductores", es decir, intermediarios, así como con los locatarios que vendían sus productos en el mercado público de esta ciudad. Uno de los requisitos que mencionan para la inclusión de productores es que se identifiquen como tales, esto es, rechazan la presencia de comerciantes en el mercado 
Héctor B. Fletes Ocón

Guadalupe Ocampo Guzmán

Guillermo Valdiviezo Ocampo
Reestructuración de la agricultura

e inseguridad alimentaria...

donde realizan la venta directa a consumidores. Igualmente, se recibe $\mathrm{y}$ vende el producto de productores que no son miembros del grupo. Pueden ingresar vendedores externos, pero para ello le piden una cuota por reja o caja de producto, y que su venta sea sólo al mayoreo.

\section{El problema de la vulnerabilidad alimentaria}

Aunque existe una variedad de prácticas y espacios de acción por parte de los pequeños productores y campesinos, es importante considerar las limitaciones que perduran en términos de seguridad alimentaria. En la Tabla 2 se muestra el patrón de minifundismo — promedio menor a 5 ha en general, pero sólo 1.9 ha en maíz-, que si bien representa una mecanismo para un nivel mínimo de abastecimiento de alimentos en unidades que producen en parte para autoconsumo (88.6\%), su situación refleja un grado de vulnerabilidad importante si tomamos en cuenta tanto la inestabilidad de las lluvias asociada con el fenómeno climático de El Niño, como la extremadamente baja proporción de hogares cuyos miembros tienen una ocupación asalariada. Es importante también observar que la estructura productiva de alimentos está muy poco diversificada, con un promedio de 2.2 cultivos por unidad. Todo esto se refleja en el bajo monto semanal de gasto en alimentos realizado por los hogares, así como en la manifestación de la presencia de problemas para obtener alimentos suficientes. En contraparte, es abrumadora la presencia de la aplicación de agroquímicos, en particular el glifosato, que se encuentra prohibido ya en algunos países. Cabe señalar que las familias que perciben problemas de inseguridad alimentaria se encuentran tanto en zonas de temporal como de riego. En las últimas el problema se asocia con la dependencia que perciben respecto de las empresas proveedoras de semillas y agroquímicos.

Es decir, aunque a través de los casos revisados la producción y posición "competitiva" de la región ha mejorado, lo que ha permitido un aumento relativo de los ingresos de los actores involucrados, esto no se da sin problemas sociales y ambientales, los cuales deben tomarse en consideración para una agenda política. En el aspecto social, la información existente nos deja ver que este tipo de reestructuración reconcentra la tierra y los recursos naturales — por ejemplo, el recurso 
Héctor B. Fletes Ocón

Guadalupe Ocampo Guzmán

Guillermo Valdiviezo Ocampo

agua en el cultivo de tomate- en algunos agricultores más privilegiados. Por el lado ambiental, en ocasiones se detecta una situación de intensificación agrícola basado en el uso de riego y agroquímicos con impactos ampliamente conocidos, como abatimiento de los mantos freáticos, contaminación de ríos y embalses —el caso de Las Lagunas de Montebello se ha convertido ya en un problema de atención política-, daños a la salud de los trabajadores y proliferación de nuevas plagas, que a la larga eleva los costos de producción. Así pues, se sugiere orientar este tipo de transformaciones en un sentido de sustentabilidad económica, social y ambiental, y no sólo económica — rentabilidad, competitividad-, que sigue siendo prioridad en las políticas de desarrollo social y agrícola en México.

\section{Reflexiones finales}

Históricamente, la agricultura se encuentra entre las mayores prioridades de los Estados, éstos instrumentan una constante e intensa regulación sobre ella. La seguridad alimentaria es y ha sido una política de Estado permanente en los diferentes países, que han tenido como ejemplos a Estados Unidos y la Unión Europea. En ese sentido, el grado en que los Estados asumen esta posición depende de las relaciones políticas y de poder que se ejercen en su interior, y de los principios y agenda que de ello deriven. En este caso, México ha preferido la importación de alimentos, que se suponía resultaría más barato que su producción, sin embargo ante la subida e inestabilidad de sus precios, tenemos los resultados ya señalados.

Resultan de gran relevancia las iniciativas que realizan los pequeños productores campesinos, como muestra el caso de esta región que está sufriendo una transformación radical en las condiciones sociales y agrarias de la población, en la lógica de la agricultura y en los recursos bióticos que se manejan. Sin embargo, al establecer nuevos cultivos distintos a granos, los campesinos se exponen a los vaivenes del mercado. A pesar de la rentabilidad que significan en el corto y mediano plazo, tienen un impacto en la degradación de los recursos naturales, dado el paquete tecnológico intensivo con el que se asocian de alto uso de agroquímicos y extracción de agua subterránea. 
Mientras, existen estrategias activas de los campesinos quienes conservan una fracción aunque sea reducida de maíz para la producción de tortilla de calidad, así es necesario revitalizar su producción, también establecer proyectos específicos de agregación de valor — dado que el nivel de ingresos es reducido y su poder adquisitivo se estanca cada vez más-, como miel o productos deshidratados de distintas frutas de la región. Todo alentado a través del fortalecimiento de las relativamente pocas organizaciones productivas que existen, las cuales dan cuenta de la importancia del tejido social en estos ámbitos rurales - la propia política nacional neoliberal de las últimas décadas ha debilitado a estas organizaciones sociales, en sus distintos niveles.

Por lo mencionado, se requiere una estrategia nacional de seguridad y soberanía alimentaria que reconozca el valor multidimensional de la agricultura y de la producción de alimentos que realizan los campesinos. La política de desarrollo agrícola actual (Programa Sectorial de Desarrollo Agropecuario, Pesquero y Alimentario 2013-2018, DOF, 2013) tiene un enfoque en los pequeños productores, pero adolece de una observación y reconocimiento de la importancia agroecológica de las prácticas de cultivo y conservación de biodiversidad que ellos desarrollan. Da prioridad a los aspectos de productividad, eficiencia por mayor escala, y seguridad alimentaria. Sin embargo, no aparecen dimensiones discutidas en este documento, que son a su vez relevantes desde la perspectiva de los pequeños productores, como: la equidad distributiva, la agroecología en el sector de PP, y la soberanía alimentaria.

$\mathrm{El}$ aumento de los ingresos agrícolas y rurales, y de la productividad, en unidades menores de 5 hectáreas — que son la mayoría—, no aparecen entre los indicadores del programa. Es importante cuestionar las políticas basadas en una falsa bipolaridad entre agricultura industrial de exportación y agricultura de subsistencia, puesto que ambas mantienen conexiones, pero en un marco de grandes asimetrías; principalmente existe una diversidad de agriculturas que hay que revalorizar y estimular. 
Héctor B. Fletes Ocón

Guadalupe Ocampo Guzmán

Guillermo Valdiviezo Ocampo

\section{Bibliografía citada}

Calva, José L., 2012, "Políticas agropecuarias para la soberanía alimentaria y el desarrollo sostenido con equidad", en José Luis Calva (coordinador), Políticas agropecuarias, forestales y pesqueras. Análisis estratégico para el desarrollo, vol. 9, Consejo Nacional de Universitarios/Juan Pablos, México, pp. 67-92.

Coneval, 2016, en http://www.coneval.org.mx/_layouts/15/xlviewer. aspx?id=/Informes/ Descarga/Anexo_estadistico.xlsx\&Default ItemOpen=1 (varias fechas de consulta).

Diario Oficial de la Federación, Programa Sectorial de Desarrollo Agropecuario, Pesquero y Alimentario 2013-2018, 13 de diciembre de 2013.

Edelman, Marc, 2014, "Food sovereignty: forgotten genealogies and future regulatory challenges", The Journal of Peasant Studies, vol. 41, núm. 6, pp. 959-978.

FAO, FIDA y PMA, 2015, El estado de la inseguridad alimentaria en el mundo 2015. Cumplimiento de los objetivos internacionales para 2015 en relación con el hambre: balance de los desiguales progresos, FAO, Roma.

Fletes, Héctor, 2013, Construyendo la globalización. Estado, mercado y actores de las cadenas agroindustriales de mango desde Chiapas, Conacyt/Unach/Ediciones de la noche, México.

Fletes, Héctor, Alejandro Macías y Jesús Madera (coordinadores), 2014a, El papel de los pequeños productores en la agricultura y alimentación. La experiencia desde tres regiones agricolas en México, Plaza y Valdés, México.

Fletes-Ocón, Héctor y Alessandro Bonanno, 2013, "Responses to the crisis of neoliberal globalization: State intervention in palm oil production in Chiapas, Mexico", International Journal of Sociology of Agriculture and Food, vol. 20, núm. 3, pp. 313-334.

Fletes Héctor, Guadalupe Ocampo, Marco Sánchez, 2014b, “Between food security and food sovereignty. Contradictory paths in Southern México", Ponencia en 113th Anual Meeting of the American Anthropological Association "Producing Anthropology", December 3-7, Washington, D. C., USA. 
Fletes, Héctor y Katia Lozano (coordinadores), 2015, Transformaciones y resistencias hacia nuevas perspectivas de desarrollo rural, AMER/ UNAM/UAM/Unach/UAN/UMSNH, México.

Gómez, Tlillalcápatl, 2013, "Redes sociales y desarrollo de innovaciones: una aproximación a localidades de la Meseta Comiteca", Revista Mexicana de Agronegocios, vol. XVII, pp. 290-304.

González, Humberto, 2013, "Especialización productiva y vulnerabilidad agroalimentaria en México", Revista Comercio Exterior, vol. 63, núm. 2, marzo y abril, pp. 21-36.

González, Humberto y Alejandro Macías, 2007, "Vulnerabilidad alimentaria y política agroalimentaria en México", Desacatos, núm. 25, pp. 47-78.

Herrera, Francisco, 2016, Procesos de reestructuración agrícola en la región fronteriza de Chiapas, Tesis de Maestría en Desarrollo Local, Unach, México.

Holt-Giménez, Eric y Raj Patel, 2012, iRebeliones alimentarias. La crisis y el hambre por la justicia!, UAZ/Miguel Ángel Porrúa, México.

Instituto de Comunicación Social del Estado de Chiapas, 2016, en http://www.icosochiapas.gob.mx/2016/08/17/se-beneficia-aproductores-de-chiapas-con-la-entrega-de-23-mil-arboles-frutales [consultado el 19 de agosto de 2016].

Lechuga, Jesús, 2006, "Capítulo III. Liberalización de la agricultura y acuerdos comerciales 1990-2000", en La estructura agraria de México: Un análisis de Largo Plazo, Biblioteca de Ciencias Sociales y Humanidades, Serie de Economía, UAM Azcapotzalco, México, D. F., pp. 95-123.

Linck, Thierry, Esteban Barragán y Hermilio Navarro, 2014, "Hacia una economía de la patrimonialización. Las apropiaciones colectivas de lo biótico y lo cognitivo", en Humberto González y Margarita Calleja (editores), Dinámica territorial agroalimentaria en tiempos de glocalización, UdeG, México, pp. 69-97.

McMichael, Philip, 2015, Regimenes alimentarios y cuestiones agrarias, UAZ/Miguel Ángel Porrúa, México. 
Moreno-Brid, Juan y Jaime Ros, 2010, Desarrollo y crecimiento en la economía mexicana. Una perspectiva histórica, FCE, México.

Nigh, Ronald, 2010, "Construcción de redes en la cadena de valor agroalimentaria. La agricultura campesina en Chiapas del siglo XXI", en Héctor Fletes (editor), Pequeños productores y vulnerabilidad global agroalimentaria, Unach/RISHORT, México

Otero, Gerardo, 2014, "El régimen alimentario neoliberal y su crisis: Estado, agroempresas multinacionales y biotecnología”, en Gerardo Otero (coordinador), La dieta neoliberal. Globalización y biotecnología agrícola en las Américas, Simon Fraser University/ UAM-X/M. A. Porrúa, México, pp. 15-42.

Otero, Gerardo, Gabriela Pechlaner, Efe Can Gürcan, 2013, “The Political Economy of 'Food Security' and Trade: Uneven and Combined Dependency”, Rural Sociology, vol. 78, núm. 3, pp. 263-289.

Puyana, Alicia y José Romero, 2008, "El sector agropecuario mexicano: un decenio con el Tratado de Libre Comercio de América del Norte. Efectos económicos y sociales", en Alicia Puyana y José Romero (coordinadores), El sector agropecuario y el Tratado de Libre Comercio de América del Norte. Efectos económicos y sociales, El Colegio de México, México, pp. 31-66.

Robles, Héctor, 2012, "El papel central de los pequeños productores en una nueva estrategia de desarrollo rural", en José Luis Calva (coordinador), Politicas agropecuarias, forestales y pesqueras. Análisis estratégico para el desarrollo, vol 9, Consejo Nacional de Universitarios/Juan Pablos Editor, México, pp. 95-115.

Robles, Héctor, 2013, "Los pequeños productores y la política pública", en Julio Moguel (coordinador), El Sur-Sureste mexicano: crisis y retos, CESOP/Cámara de Diputados, LXII Legislatura/ Juan Pablos Editor, México, pp. 81-117.

Rodríguez-Gómez, Guadalupe, 2013, “The Debate Over Food Sovereignty in México”, Food Sovereignty: A Critical Dialogue, International Conference, September 14-15, Yale University. 
Rubio, Blanca, 2014, El dominio del hambre. Crisis de hegemonía y alimentos, Universidad Autónoma Chapingo, México.

SIAP, Sistema de Información Agroalimentaria y Pesquera, 2016, en http://www.gob.mx/siap/acciones-y-programas/produccionagricola-33119?idiom $=$ es

Tetreault, Darcy, 2012, "Propuestas campesinas para la soberanía alimentaria de México", en José Luis Calva (coordinador), Políticas agropecuarias, forestales y pesqueras. Análisis estratégico para el desarrollo, vol 9, Consejo Nacional de Universitarios/Juan Pablos Editor, México, pp. 116-137.

Turrent, Antonio, Timothy Wise y Elise Garvey, 2012, Factibilidad de alcanzar el potencial productivo de maiz, Mexican Rural Development Research Reports, núm. 24, Woodrow Wilson International Center for Scholars.

Villafuerte, Daniel, 2015, "Crisis rural, pobreza y hambre en Chiapas", Revista LiminaR. Estudios Sociales y Humanísticos, vol. XIII, núm. 1, México, pp. 13-28.

Weisbrot, Mark, Stephan Lefebvre y Joseph Sammut, 2014, ¿El TLCAN ayudó a México? Una valoración tras 20 años, Center for Economic and Policy Research. 